\title{
IFN $\beta$ enhances mesenchymal stromal (Stem) cells immunomodulatory function through STAT1-3 activation and mTOR- associated promotion of glucose metabolism
}

Tiziana Vigo ${ }^{1}$, Claudia La Rocca ${ }^{2}$, Deriggio Faicchia ${ }^{2}$, Claudio Procaccini ${ }^{2,3}$, Maddalena Ruggieri ${ }^{4}$, Marco Salvetti ${ }^{5,6}$, Diego Centonze ${ }^{6,7}$, Giuseppe Matarese ${ }^{2,8}$ and Antonio Uccelli, ${ }^{1,9}$, on behalf of the MSRUN Network

\begin{abstract}
Administration of mesenchymal stem cells (MSC) ameliorate experimental autoimmune encephalomyelitis (EAE), a mouse model of multiple sclerosis (MS), at both clinical and neuropathological levels. The therapeutic properties of MSC in EAE are mainly mediated by the modulation of pathogenic immune response, but other neurotropic effects, including decreased demyelination and axonal loss as well as promotion of tissue repair, play also a role. Properly controlled phase II clinical trials to explore the potential of MSC transplantation as a treatment for MS are underway. Interferon beta (IFN $\beta$ ) is an approved treatment for relapsing-remitting and secondary progressive MS. Here, we explored the possibility that IFN $\beta$ might influence the therapeutic potential of MSC, in view of possible synergistic effects as add-on therapy. IFN $\beta$ enhanced the immunomodulatory functions of MSC and induced the expression of secretory leukocyte protease inhibitor (S/pi) and hepatocyte growth factor (Hgf), two soluble mediators involved in immune and regenerative functions of MSC. At molecular level, IFN $\beta$ induced a rapid and transient phosphorylation of STAT1 and STAT3, the transcription factors responsible for Slpi and Hgf induction. Concomitantly, IFN $\beta$ dynamically affected the activity of mTOR, a key checkpoint in the control of metabolic pathways. Indeed, the impairment of mTOR activity observed early upon exposure to IFN $\beta$, was followed by a longlasting induction of mTOR signaling, that was associated with an increased glycolytic capacity in MSC. When induced to switch their energetic metabolism towards glycolysis, MSC showed an improved ability to control T-cell proliferation. These results suggest that modifications of MSC energetic metabolism induced by IFN $\beta$ may contribute to promote MSC immunomodulatory function and support a role for metabolic pathways in the therapeutic function of MSC. Altogether, these findings support the idea of a combined treatment for MS, in which the immunomodulatory and possibly regenerative activity of MSC could be enhanced by the administration of IFN $\beta$.
\end{abstract}

Correspondence: Antonio Uccelli (auccelli@neurologia.unige.it)

${ }^{1}$ IRCCS Ospedale Policlinico San Martino, Genoa, Italy

${ }^{2}$ Laboratorio di Immunologia, Istituto di Endocrinologia e Oncologia

Sperimentale, Consiglio Nazionale delle Ricerche (IEOS-CNR), Naples, Italy

Full list of author information is available at the end of the article.

Edited by G. Melino

\section{Introduction}

Mesenchymal Stromal (Stem) Cells (MSC) are a heterogeneous subset of stromal progenitors of mesodermal lineage that have been isolated from almost every tissue, mainly the bone marrow and adipose tissue. MSC are defined on the basis of their capability to grow as adherent

\section{(c) The Author(s) 2019}

(c) (i) Open Access This article is licensed under a Creative Commons Attribution 4.0 International License, which permits use, sharing, adaptation, distribution and reproduction cc) in any medium or format, as long as you give appropriate credit to the original author(s) and the source, provide a link to the Creative Commons license, and indicate if changes were made. The images or other third party material in this article are included in the article's Creative Commons license, unless indicated otherwise in a credit line to the material. If material is not included in the article's Creative Commons license and your intended use is not permitted by statutory regulation or exceeds the permitted use, you will need to obtain permission directly from the copyright holder. To view a copy of this license, visit http://creativecommons.org/licenses/by/4.0/. 
cells on plastic, to display a fibroblast-like morphology, to form colonies in vitro supporting hematopoiesis, to differentiate into cells of the mesodermal lineage and express stromal while lacking hematopoietic markers ${ }^{1}$. Several studies showed that MSC possess immunomodulatory properties exerted on cells populations of both adaptive and innate immunity ${ }^{2}$ and these features, together with their reported ability to protect neural cells from death and foster neural repair, account for their proposed therapeutic effect on multiple sclerosis (MS) and other neurological diseases ${ }^{3}$. Intravenous infusion of MSC improved the clinical course of EAE, inducing immune tolerance, reducing inflammation decreasing demyelination and promoting tissue repair ${ }^{4-9}$. The mechanisms through which MSC exert their therapeutic function are heterogenous and probably pleiotropic. It is generally accepted that MSC immunomodulation is strongly influenced by cytokines in the inflammatory environment, particularly by IFN gamma $(\operatorname{IFN} \gamma)^{10,11}$ and that their therapeutic effect is mediated by paracrine mechanisms through the release of soluble factors ${ }^{2}$. Particularly, intravenous injection of conditioned medium containing MSC-derived hepatocyte growth factor (HGF) promoted remyelination in vitro and tissue repair in vivo ${ }^{12}$. Autologous MSC have been safely administrated in a limited number of patients with $\mathrm{MS}^{13}$, and ongoing controlled clinical studies to explore the potential of MSC transplantation as a treatment for MS have been launched (Clinical Trial NCT01854957).

Interferon beta (IFN $\beta$ ) is an approved treatment for relapsing-remitting ${ }^{14}$ and secondary progressive multiple sclerosis (MS). Interferons are a family of cytokines secreted by various cell types of the innate and adaptive immune systems as well as by other tissues. One major pathway in IFN $\beta$ signaling involves activation of Signal Transducer and Activator of Transcription (STAT) proteins and formation of complexes that translocate to the nucleus and bind to specific elements to regulate gene transcription ${ }^{15}$. Efficacy of IFN $\beta$ for the treatment of MS is believed to be due to modulation of immune responses ${ }^{16}$. Among its functions, in MS subjects IFN $\beta$ modulates dendritic cells ${ }^{17}, T$ and $B$ lymphocytes $^{18}$, as well as regulatory NK cells and $\mathrm{T}$ regulatory cells ${ }^{19}$.

While the potent immunomodulatory effect of IFN $\beta$ on cells of the immune system has been extensively studied, little is known on the interaction between IFN $\beta$ and MSC. We recently demonstrated that the pro-immunomodulatory effect of IFNY on MSC is mediated by the phosphorylation of STAT1 and STAT3 and by the inhibition of mammalian target of rapamycin (mTOR) activity ${ }^{11}$. Thus, we sought to address the effect of IFN $\beta$ on MSC immunomodulatory functions keeping in mind that, based on the possibility of a synergic effect, these two treatments could be effectively associated to treat MS.

Our data showed that IFN $\beta$ promoted the ability of MSC to control T-cell proliferation and enhanced the gene expression of $H g f$ and of secretory leukocyte protease inhibitor (Slpi), an important regulator of innate and adaptive immunity and a component of tissue regenerative $\operatorname{programs}^{20}$. At molecular level IFN $\beta$ induced a rapid activation of STAT1 and STAT3 in MSC, concomitant with an early inhibition followed, at $24 \mathrm{~h}$, by a strong reactivation of mTOR pathway. The oscillatory switch of mTOR signaling induced by IFN $\beta$ was associated with an increased glycolytic capacity of MSC, that improved their ability to control T-cell proliferation.

\section{Materials and methods \\ MSC culture and treatments}

MSC isolated from mouse bone marrow were cultured in Mesencult medium as described [8]. MSC were treated with $1500 \mathrm{UI} / \mathrm{ml}$ of IFN $\beta$ (Rebif ${ }^{\circledR} 44$, Merk Serono) for 1 $h$ in serum-free RPMI (Gibco).

\section{Gene silencing}

siRNA transfection was carried out using Lipofectamine 2000 in serum free RPMI for $24 \mathrm{~h}$ in the presence of specific siRNA (20 pmol). STAT1:(gauugaccuggagaccaccucucuu/ aagagagguggucuccaggucaauc)

SLPI: (caagugcugugaggguaua $(\mathrm{dt})(\mathrm{dt}) /$ uauacccucacagca$\operatorname{cuug}(\mathrm{dt})(\mathrm{dt})$

As negative control, MSC were transfected with AllStar siRNA (Qiagen) (CTRL-KD) according to manufacturer's instructions. The efficacy of siRNA knock-down was tested by real time PCR.

\section{Collection of MSC-conditioned medium}

Confluent MSC were primed with IFN $\beta$ for $1 \mathrm{~h}$ in serum-free RPMI. MSC were washed and cultured for an additional $24 \mathrm{~h}$ in serum-free RPMI with glucose 2 or $4.5 \mathrm{~g} / \mathrm{L}$, and the medium was collected (conditioned medium, CM).

\section{In vitro T-cell proliferation assay}

MSC CM equivalent to that of $2 \times 10^{4}$ MSC was assessed for its effect on proliferation of $2 \times 10^{5}$ anti-CD3/CD28activated spleen cells (1:10 ratio) labeled with CFSE $(1 \mu \mathrm{M}$; Molecular Probes). Equivalent amount of CM from 3T3 fibroblast culture was used as control. Where the CM of MSC was collected in RPMI with different concentrations of glucose ( 2 or $4.5 \mathrm{~g} / \mathrm{L})$, the final glucose concentration in the T-cell activation medium was $4.5 \mathrm{~g} / \mathrm{L}$. Alternatively, MSC CTRL-KD and SLPI-KD were irradiated (5000 rad) and added to $2 \times 10^{\wedge} 5$ anti-CD3/CD28-activated spleen cells (1:10 ratio) labeled with CFSE $(1 \mu \mathrm{M})$ at the ratio of 
1:40. After $72 \mathrm{~h}$, splenocytes were collected and the mean fluorescence intensity of CD3-positive T cells was evaluated by flow cytometry. Statistical differences were evaluated using Student's $T$-test.

\section{Real-time PCR}

Total RNA was extracted with Trizol (Invitrogen) from MSC, MSC RAPA, MSC CUC, MSC CTRL-KD and STAT1-KD $24 \mathrm{~h}$ after treatment with IFN $\beta$. Real-time PCR experiments were conducted as per the manufacturer's instructions (SYBR Green I Master, Roche) using the following primers:

Cd274 (aaatcgtggtccccaagc/tcctcatgttttgggaactatct)

$\mathrm{Ccl} 2$ (catccacgtgttggctca/gatcatcttgctggtgaatgagt)

$\mathrm{Hgf}$ (caccccttgggagtattgtg/gggacatcagtctcattcacag)

Il18bp (agctattcggggcttaggag/tgcaagcaagtctggtgtct)

Slpi (cttgctctggggatcctg/ggctccgattttgatagcat)

Gene expression values were calculated as $2^{(-\Delta C T)}$ with hypoxanthine guanine phosphoribosyl transferase (Hprt) (tcctcctcagaccgctttt/cctggttcatcatcgctaatc) as reference gene. Statistical differences were evaluated using Student's $T$-test.

\section{Western blot}

Total cell lysates and western blot analysis were performed as previously described ${ }^{21}$. The blots were probed with antibodies specific for pSTAT1 (Tyr701), pSTAT3 (Tyr705), pS6 (all 1:1000 dilution and from Cell Signaling Technology, Beverly, MA) and with an actin antibody (1:1000 from Santa Cruz) to normalize for the amount of loaded protein.

\section{Pathway inhibition by pharmacological agents}

STAT3 inhibition was obtained by exposure of MSC to cucurbitacin I (CUC) ( $500 \mathrm{nM}$; Sigma Aldrich) for $3 \mathrm{~h}$ in serum-free RPMI; mTOR inhibition was obtained through exposure of MSC to rapamycin ( $100 \mathrm{nM}$; Sigma Aldrich) in serum-free RPMI for $1 \mathrm{~h}$ (RAPA). The inhibition of STAT3 and S6 phosphorylation following treatments was evaluated by Western blot analysis using phospho-specific antibodies (data not shown).

\section{Immunofluorescence}

MSC were fixed in 4\% paraformaldehyde, permeabilized with $0.5 \%$ Triton and labeled with antibodies specific for mouse SLPI (1:100 dilution from Cell Signaling) followed by a secondary antibody conjugated with AlexaFluor-594. Nuclei were stained with DAPI. The slides were analyzed with a Olympus microscopy equipped with 405 and 561 nm filters.

\section{Analysis of MSC metabolism}

Real-time measurements of extracellular acidification rate (ECAR) and oxygen consumption rate (OCR) were measured using an XFe-96 Extracellular Flux Analyzer (Seahorse Bioscience). Cells were counted using an automated Cell counter (Countess from Life Technologies), seeded in XFe-96 plates (Seahorse Bioscience) at the density of $2 \times 10^{4}$ cells/well and incubated overnight at $37{ }^{\circ} \mathrm{C}$ in $5 \% \mathrm{CO}_{2}$ atmosphere in the presence or absence of IFN $\beta(1500 \mathrm{UI} / \mathrm{ml})$. ECAR was measured in XF media in basal condition and in response to $10 \mathrm{mM}$ glucose, $4 \mu \mathrm{M}$ oligomycin and $100 \mathrm{mM}$ of 2-Deoxy-D-glucose (2-DG). Basal glycolysis was calculated after glucose injection (subtracting the ECAR rate inhibited by 2DG). Maximal glycolysis was measured after oligomycin injection and glycolytic capacity as the difference of oligomycininduced ECAR and 2-DG-induced ECAR. OCR was measured in XF media (non-buffered DMEM medium, containing $10 \mathrm{mM}$ glucose, $2 \mathrm{mM} \mathrm{L}$-glutamine, and $1 \mathrm{mM}$ sodium pyruvate), under basal conditions and in response to $5 \mu \mathrm{M}$ oligomycin, $1.5 \mu \mathrm{M}$ of carbonylcyanide-4-(trifluoromethoxy)-phenylhydrazone (FCCP) and $1 \mu \mathrm{M}$ of antimycin A and rotenone (all chemicals from Sigma Aldrich). Basal OCR was calculated as the difference between baseline measurements and antimycin A/rotenone-induced OCR; maximal OCR was measured as the difference between FCCP-induced OCR and antimycin A/rotenone-induced OCR. Finally, the ATP-linked parameter was calculated as the difference between baseline measurements, without injection, and oligomycininduced OCR. Experiments with the Seahorse system were done with the following assay conditions: $3 \mathrm{~min}$ mixture; $3 \mathrm{~min}$ wait; and $3 \mathrm{~min}$ measurement. Then, metabolic parameters were calculated. Data are expressed as mean and S.E.M. from four independent experiments. Statistical differences were evaluated using the Wilcoxon matched-pairs test.

\section{Results \\ IFN $\beta$ promoted MSC immunomodulation inducing the expression of secreted mediators}

To verify the possibility that IFN $\beta$ may affect the expression of immunomodulatory factors secreted by MSC we analyzed, $24 \mathrm{~h}$ upon priming with IFN $\beta$, the mRNA levels of $C d 274, C c l 2$, and $H g f$, which are known to act in a paracrine way on immune cells. We also assessed the gene expression of Il18bp and Slpi, that we recently identified as induced in MSC upon interaction with activated $\mathrm{T}$ cells ${ }^{11}$. As shown Fig. 1a, IFN $\beta$ induced a significant increase of mRNA level of all the genes analyzed. We then focused on Slpi, whose role in MSC immunomodulation has never been reported. We first confirmed that MSC exposed to IFN $\beta$ increased Slpi expression at protein level (Fig. 1b). To get some insight into the role of Slpi on MSC immunoregulatory function, we reduced its expression in MSC through gene silencing (SLPI-KD) and used these Slpi-silenced cells to inhibit 


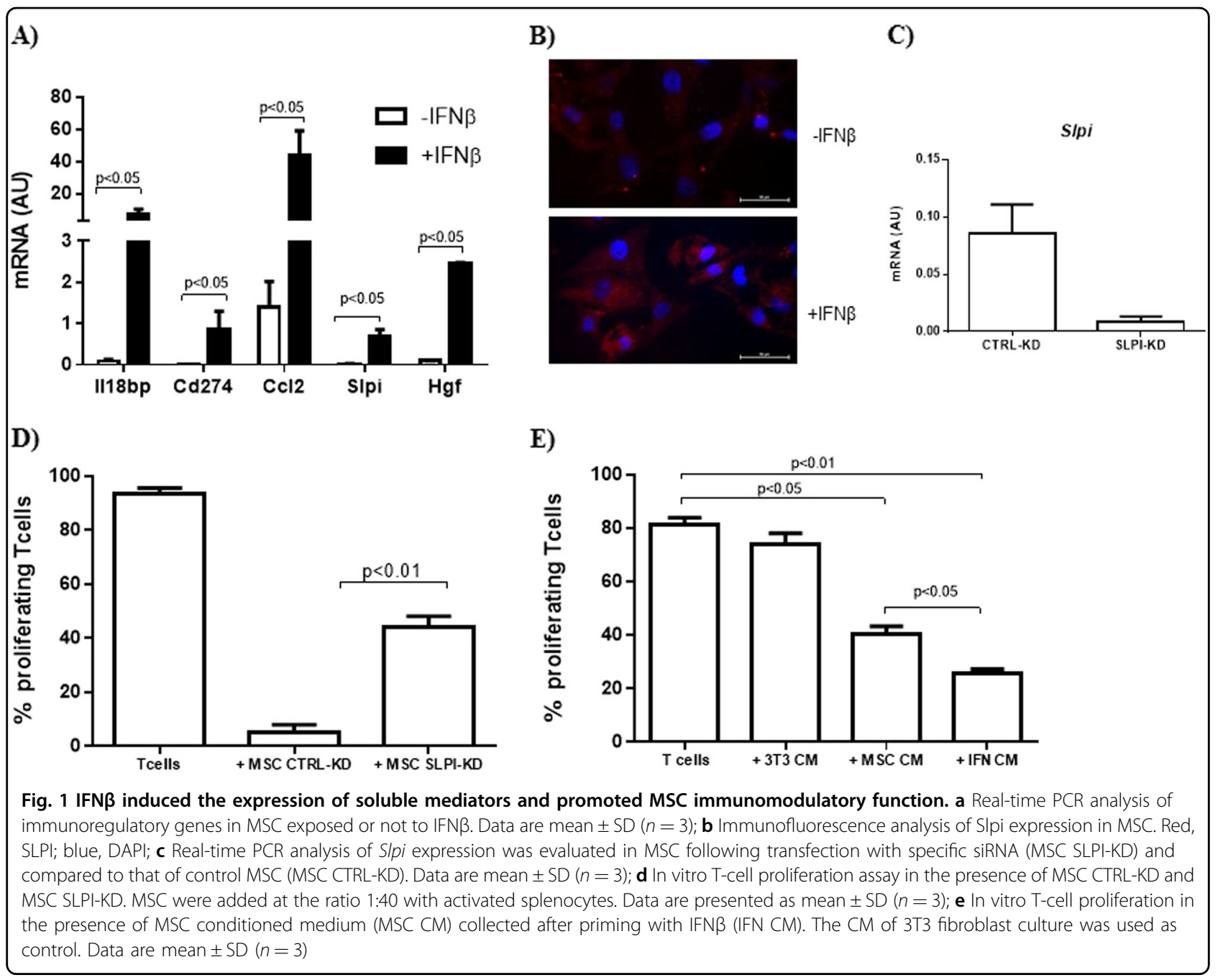

T-cell proliferation (Fig. 1c). As shown in Fig. 1d, MSC SLPI-KD showed a reduced ability to inhibit T-cell proliferation in vitro as compared to MSC transfected with control siRNA (CTRL-KD).

Then we sought to verify if IFN $\beta$ was able to affect the ability of MSC to control T-cell proliferation priming MSC with IFN $\beta$ and testing the effect of the derived conditioned medium (CM) on activated T cells. As shown in Fig. 1e, IFN $\beta$ significantly enhanced the constitutive immunomodulatory effect of MSC CM.

These results demonstrated that IFN $\beta$ induced in MSC the expression of soluble immunomodulatory factors and concomitantly improved their ability to inhibit T-cell proliferation in vitro.

IFN $\beta$ induced dynamic changes in STAT1, STAT3, and S6 phosphorylation

As activation of STAT1 and STAT3, and inhibition of mTOR pathway positively affects MSC immunomodulatory function upon administration of interferon gamma
$(\mathrm{IFN} \gamma)^{11}$, we analyzed the activation state of these pathways following exposure to IFN $\beta$. Analysis of phosphorylated pathway components revealed that exposure to IFN $\beta$ for $1 \mathrm{~h}$ dramatically increased the levels of phosphorylated STAT1 (pSTAT1) and STAT3 (pSTAT3) and concomitantly reduced the amount of phosphorylated S6 (pS6), used as read-out of mTOR activity (Fig. 2b). Upon a prolonged exposure $(24 \mathrm{~h}), \mathrm{pSTAT} 1$ and pSTAT3 decreased as compared to $1 \mathrm{~h}$, indicating that IFN $\beta$ induced a a transient phosphorylation of STAT1 and STAT3. Concomitantly, the levels of pS6 strongly increased as compared to that observed upon exposure to IFN $\beta$ for $1 \mathrm{~h}$, revealing that IFN $\beta$ induced an oscillatory modification of mTOR pathway activity (Fig. 2a, b).

\section{IFN $\beta$-dependent expression of Slpi and $\mathrm{Hgf}$ was mediated by STAT1 and STAT3}

Engagment of STAT1 and STAT3 induces the expression of immunomodulatory genes in MSC, that is further enhanced by inhibition of mTOR signaling ${ }^{11}$. To assess 

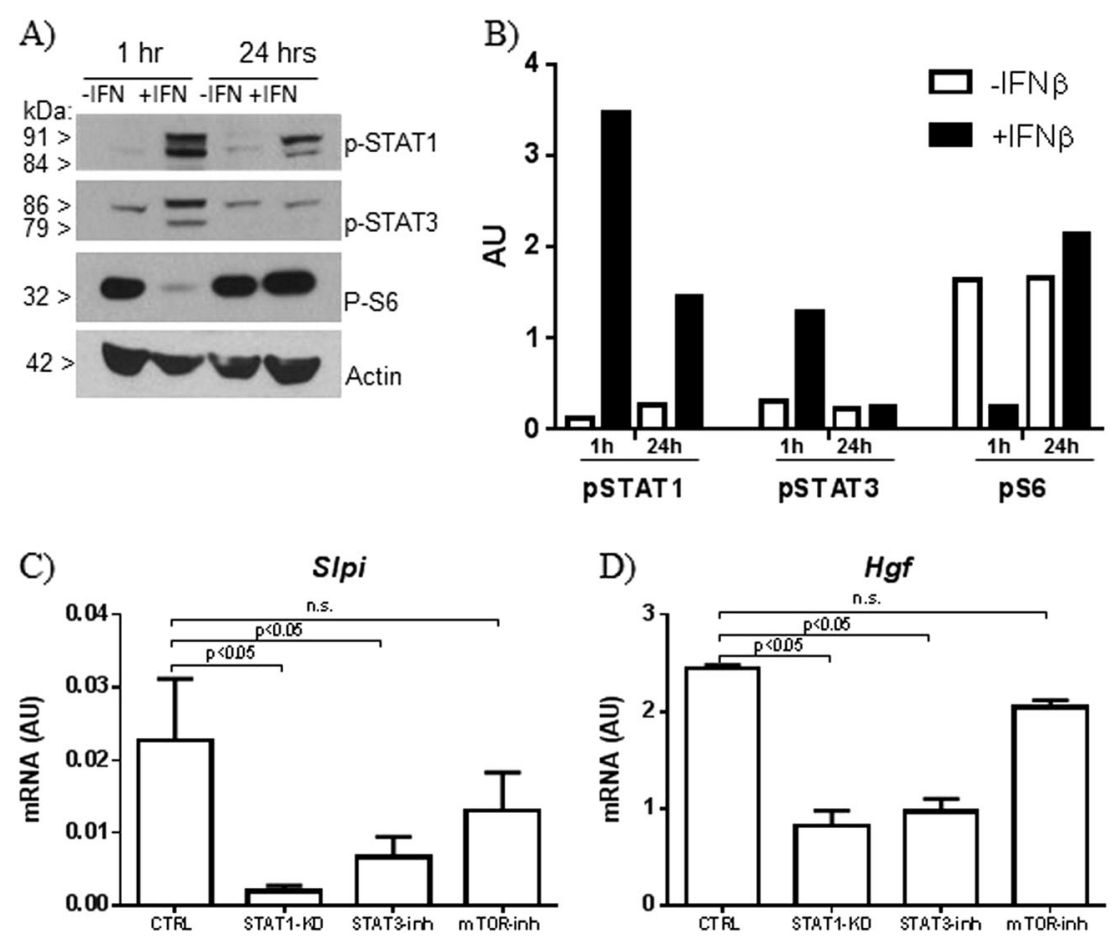

Fig. 2 IFN $\beta$ induced dynamic changes in STAT1, STAT3, and S6 phosphorylation. a Western blot analysis conducted after 1 or $24 \mathrm{~h}$ of incubation with IFNß. Actin served as loading control; $\mathbf{b}$ relative densitometric quantitation of the shown gel, after normalization on actin; $\mathbf{c}$, $\mathbf{d}$ Real-time PCR analysis of Slpi and Hgf expression in MSC exposed to IFN $\beta$ upon pathway inhibition. Data are presented as mean \pm SD $(n=3)$

the role of these pathways in the induction of Slpi and $H g f$ by IFN $\beta$, we inhibited STAT1 through gene silencing, STAT3 using the chemical inhibitor Cucurbitacin I (CUC) and mTOR with its inhibitor rapamycin (RAPA) before the exposure to IFN $\beta$ and we analyzed Slpi and $H g f$ expression by real-time PCR. We observed that the induction of Slpi expression by IFN $\beta$ was significantly impaired by STAT1 and STAT3 inhibition, while the blockade of mTOR pathway did not exert any affect on their expression (Fig. 2c). Similarly, we observed that impairment of STAT1 and STAT3 signaling, but not that of mTOR, reduced the induction of $H g f$ upon exposure to IFN $\beta$ (Fig. 2d).

\section{Exposure to IFN $\beta$ modified MSC energetic metabolism}

As IFN $\beta$ affected the activity of mTOR pathway, a master regulator of intracellular metabolism, we assessed whether exposure to IFN $\beta$ could impact on the bioenergetic profile of MSC, by measuring extracellular acidification rate (ECAR) as an indicator of glycolysis, and oxygen consumption rate (OCR) indicator of mitochondrial respiration. We observed that IFN $\beta$ did not modify basal glycolysis in MSC (Fig. 3a, b). However, maximal glycolysis (induced by oligomycin injection) and glycolytic capacity were significantly increased by IFN $\beta$ treatment, as compared to untreated MSC (Fig. 3a, b). Then we investigated mitochondrial respiration in terms of OCR. IFN $\beta$ did not affect maximal OCR and OCR coupled to ATP generation, but slightly enhanced the respiratory capacity of MSC in basal conditions (Fig. 3c, d), indicating that IFN $\beta$ preferentially increased the glycolytic capacity of MSC.

\section{Glycolisis engagement improved MSC immunomodulatory function}

To link MSC glucose metabolism with their immunomodulatory function, we assessed whether or not improvement of MSC glycolitic rate may impact on their capacity to inhibit T-cell proliferation. We boosted glycolitic flux by growing MSC in high glucose containing medium $(4.5 \mathrm{~g} / \mathrm{L})$, and we assessed the immunomodulatory properties of the resulting CM in T-cell proliferation assay. We obtained a significant improvement in the constitutive ability of MSC CM to inhibit T-cell proliferation by collecting MSC CM in high glucosecontaining medium as compared to MSC CM prepared in normal medium ( $2 \mathrm{~g} / \mathrm{L}$ ) (Fig. 4$)$.

These results clearly demonstrated that promoting glucose metabolism improved the immunomodulatory function of MSC, thus suggesting a new possible mechanism through which IFN $\beta$ enhances MSC immunomodulation. 


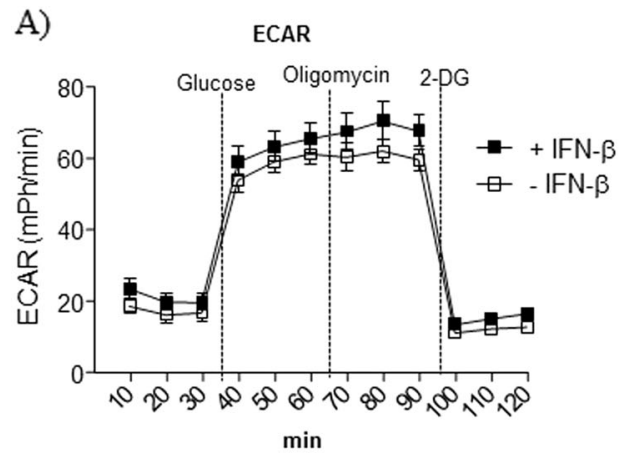

C)

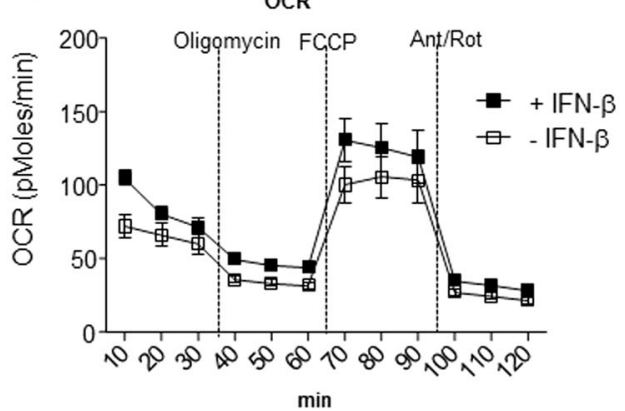

B)
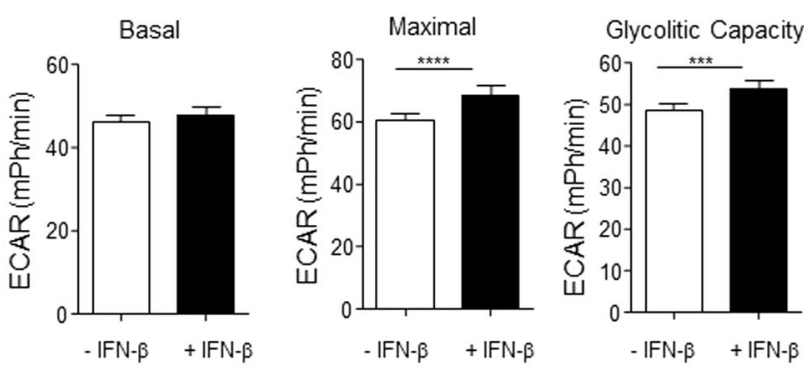

D)

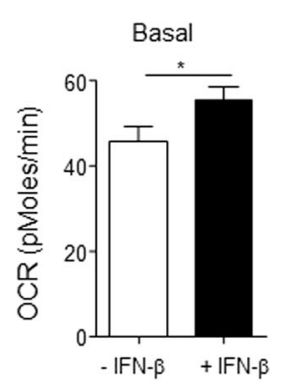

Mitochondrial Respiration

Maximal

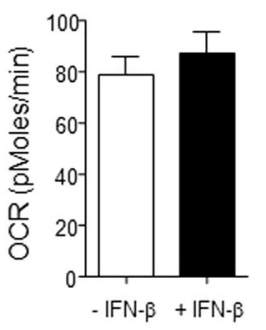

ATP linked

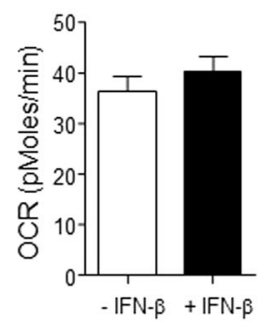

Fig. 3 Exposure to IFN $\beta$ modified MSC energetic metabolism. a Kinetic profile of ECAR in MSC treated or not with IFN $\beta$ for $12 \mathrm{~h}$. The data are shown as mean \pm S.E.M. of four independent experiments. ECAR was measured in real time, under basal conditions and in response to glucose, oligomycin and 2-DG; $\mathbf{b}$ parameters of glycolysis in MSC were calculated as detailed in Materials and methods. Data are expressed as mean \pm S.E.M. of three measurements, from four independent experiments; $\mathbf{c}$ kinetic profile of OCR in MSC treated or not with IFN $\beta$ for $12 \mathrm{~h}$. The data are shown as mean \pm S.E.M. of four independent experiments. OCR was measured in real time, under basal conditions and in response to oligomycin, FCCP and Antimycin A + Rotenone. $\mathbf{d}$ Parameters of mitochondrial respiration in MSC were calculated as detailed in material and methods. Data are expressed as mean \pm S.E.M. of three measurements, from four independent experiments

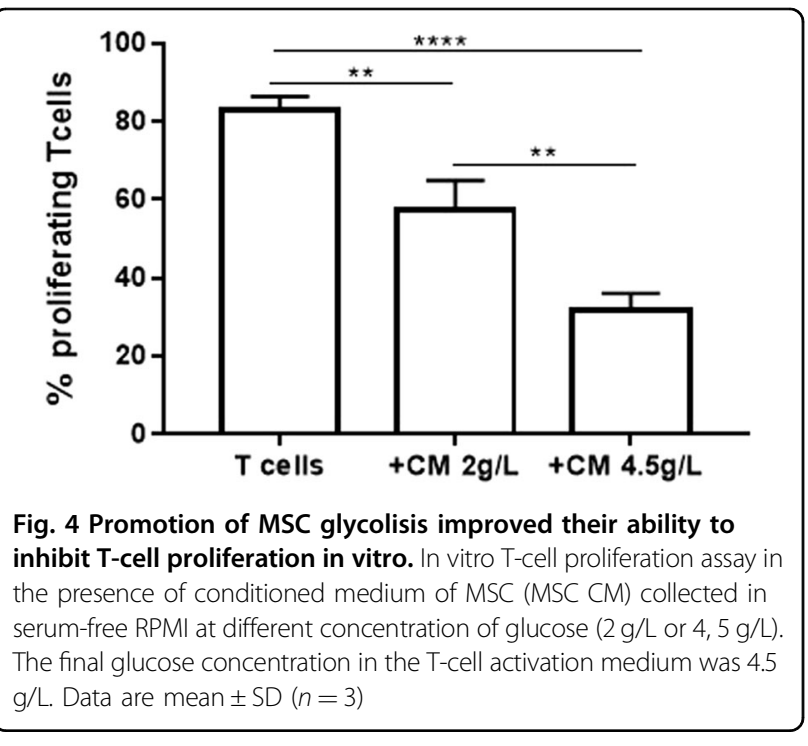

\section{Discussion}

While the potent immunomodulatory effect of IFN $\beta$ on cell types of the immune system has been extensively studied $^{22}$, little is known on the interaction between IFN $\beta$ and MSC. IFN $\beta$ was shown to influence the expression of chemokines and their receptors in human $\mathrm{MSC}^{23}$, but the effects of IFN $\beta$ on MSC immunomodulatory function are largely unknown. Our data demonstrated that IFN $\beta$ significantly increased the expression of molecules, secreted by MSC, with reported immunomodulatory activities. Among those, we observed that Slpi, a molecule never related to MSC biology, potentiated the ability of MSC to control T-cell proliferation in vitro.

Slpi is a polypeptide secreted by epithelial cells to protect tissue from damage by leucocyte proteolytic enzymes. Nevertheless, the regulatory function of Slpi on adaptive immunity is independent to its anti-protease activity. Slpi has been demonstrated to affect the monocyte pattern secretion by preventing the interaction of p 65 with the NF-kB binding sites ${ }^{24}$, to inhibit effector T-cell proliferation ${ }^{25}$ and to promote the generation of regulatory $\mathrm{T}$ cells $^{26}$. Slpi is upregulated in macrophages, activated microglia, neuronal cells and astrocytes during EAE attack $^{27}$. Interestingly, Slpi was shown to increase proliferation of adult neural stem cells promoting oligodendroglial differentiation, as incubation of adult neural stem cells with recombinant Slpi resulted in an increase of cell 
proliferation and of differentiation towards oligodendrocytes ${ }^{27}$. Whether Slpi secreted by MSC may play a role also in tissue repair promoted by MSC requires further investigation. Interestingly IFN $\beta$ significantly enhanced also the expression of Hgf a pleiotropic cytokine with neuroprotective which has been demonstrated to enhance axonal outgrowth and oligodendroglial maturation ${ }^{12}$.

At molecular level, IFN $\beta$ induced a transient phosphorylation of STAT1 and STAT3, determining the induction of Slpi and $H g f$ expression, that was indeed impaired by both STAT1 and STAT3 inhibition. Concomitantly, IFN $\beta$ induced a dynamic modification of mTOR activity, that was early reduced and then increased. In line with the role of mTOR in the control of glucose metabolism, a prolonged exposure to IFN $\beta$ increased MSC glycolytic capacity, thus improving their ability to engage glycolysis to convert glucose to pyruvate or lactate. Energy metabolism and functional activation are fully integrated in immune cells to determine their ability to divide, differentiate, and carry out effector functions ${ }^{28}$. Here we found that inhibition of T-cell proliferation by MSC was strongly enhanced by boosting MSC glycolisis. As ECAR, monitored as readout of MSC glycolitic activity, was mainly due to production of lactate, our data suggest the possible involvement of lactic acid in MSC immunomodulatory function. Lactic acid had been shown to suppress the proliferation and cytokine production of human cytotoxic $\mathrm{T}$ lymphocytes ${ }^{29}$ and to inhibit TNF secretion and glycolysis of human monocytes ${ }^{30}$.

Our data support that IFN $\beta$ can enhance MSC therapeutic function through two different molecular mechanisms: the early induction of STAT1-3-dependent immunomodulatory genes, and the long-lasting mTORassociated promotion of glucose metabolism. Particularly, based on the finding that IFN $\beta$ induced the expression of cytokines with both immunomodulatory and neuroprotective features such as Hgf and Slpi, we speculate that MSC as add-on therapy to IFN $\beta$ treatment could promote tissue repair beyond modulation of inflammatory reactions. In line with these results, administration of IFN $\beta$ secreting engineered MSC has been demonstrated to be more effective than wild-type MSC in the attenuation of $\mathrm{EAE}^{31,32}$, further supporting the rationale for combined protocols to treat MS. Moreover, the finding that MSC immunomodulatory function is improved by glucose metabolism revealed a new possible mechanism underlying MSC therapeutic effect.

\footnotetext{
Acknowledgements

This work was supported by grants from Fondazione Italiana Sclerosi Multipla (FISM) (grant MESEMS-FISM 2012/S/4 to A.U.), (grant FISM no. 2016/R/18, no. 2014/R/21 to G.M.), from the Compagnia di San Paolo (grant 2015-0646 to A.U.) and by a grant from Merck Serono SpA (Multiple Sclerosis Research University Network, MS-RUN project to A.U., M.R., M.S., D.C., and G.M.). T.V. was supported by Fondazione Italiana Sclerosi Multipla (FISM) (grant 2017/R/28). G.M. was supported by grants from European Research Council Grant
}

"menTORingTregs" no. 310496, from Fondazione Italiana Sclerosi Multipla (FISM) no. 2016/R/18 and from Telethon no. GGP17086. C.P. was supported by the Italian Ministry of Health, GR-2016-02363749. The sponsors had no role in study design, data collection and analysis, decision to publish or preparation of the paper.

\section{Author details}

${ }^{1}$ IRCCS Ospedale Policlinico San Martino, Genoa, Italy. ${ }^{2}$ Laboratorio di Immunologia, Istituto di Endocrinologia e Oncologia Sperimentale, Consiglio Nazionale delle Ricerche (IEOS-CNR), Naples, Italy. ${ }^{3}$ IRCSS Fondazione Santa Lucia, Rome, Italy. ${ }^{4}$ Department of Basic Medical Science, Neuroscience and Sense Organs, University of Bari, Bari, Italy. ${ }^{5}$ Centre for Experimental Neurological Therapies (CENTERS), Sapienza University, Rome, Italy. ${ }^{6}$ IRCCS Istituto Neurologico Mediterraneo (INM) Neuromed, Pozzilli, Italy. ${ }^{7}$ Laboratory of Synaptic Immunopathology, Tor Vergata University, Rome, Italy. ${ }^{8}$ Dipartimento di Medicina Molecolare e Biotecnologie Mediche, Università degli Studi di Napoli "Federico II", Naples, Italy. ${ }^{9}$ Department of Neurosciences, Rehabilitation, Ophthalmology, Genetics, Maternal and Child Health Unit and Center of Excellence for Biomedical Research, University of Genoa, Genoa, Italy

\section{Author contributions}

Tiziana Vigo: conception and design, collection and/or assembly of data, data analysis and interpretation, manuscript writing, final approval of manuscript. Claudia La Rocca: collection and/or assembly of data, data analysis and interpretation, final approval of manuscript. Deriggio Faicchia: collection and/ or assembly of data, data analysis and interpretation, final approval of manuscript. Claudio Procaccini: collection and/or assembly of data, data analysis and interpretation, final approval of manuscript. Maddalena Ruggieri: conception and design, final approval of manuscript. Marco Salvetti: conception and design, final approval of manuscript. Diego Centonze: conception and design, final approval of manuscript. Giuseppe Matarese: conception and design, data analysis and interpretation, manuscript writing, final approval of manuscript. Antonio Uccelli: conception and design, financial support, manuscript writing, final approval of manuscript.

\section{Conflict of interest}

The authors declare that they have no conflict of interest.

\section{Publisher's note}

Springer Nature remains neutral with regard to jurisdictional claims in published maps and institutional affiliations.

Received: 22 October 2018 Revised: 31 December 2018 Accepted: 7 January 2019

Published online: 28 January 2019

\section{References}

1. Horwitz, E. M. et al. Clarification of the nomenclature for MSC: The International Society for Cellular Therapy position statement. Cytotherapy 7, 393-395 (2005).

2. Uccelli, A., Moretta, L. \& Pistoia, V. Mesenchymal stem cells in health and disease. Nat. Rev. Immunol. 8, 726-736 (2008).

3. Uccelli, A., Laroni, A. \& Freedman, M. S. Mesenchymal stem cells for the treatment of multiple sclerosis and other neurological diseases. Lancet Neurol. 10, 649-656 (2011).

4. Bai, L. et al. Human bone marrow-derived mesenchymal stem cells induce Th2-polarized immune response and promote endogenous repair in animal models of multiple sclerosis. Glia 57, 1192-1203 (2009).

5. Gerdoni, E. et al. Mesenchymal stem cells effectively modulate pathogenic immune response in experimental autoimmune encephalomyelitis. Ann. Neurol. 61, 219-227 (2007).

6. Gordon, D. et al. Human mesenchymal stem cells abrogate experimental allergic encephalomyelitis after intraperitoneal injection, and with sparse CNS infiltration. Neurosci. Lett. 448, 71-73 (2008).

7. Rafei, M. et al. Mesenchymal stromal cells ameliorate experimental autoimmune encephalomyelitis by inhibiting CD4 Th17 T cells in a CC chemokine ligand 2-dependent manner. J. Immunol. 182, 5994-6002 (2009). 
8. Zappia, E. et al. Mesenchymal stem cells ameliorate experimental autoimmune encephalomyelitis inducing T-cell anergy. Blood 106, 1755-1761 (2005).

9. Zhang, J. et al. Human bone marrow stromal cell treatment improves neurological functional recovery in EAE mice. Exp. Neurol. 195, 16-26 (2005).

10. Ren, G. et al. Mesenchymal stem cell-mediated immunosuppression occurs via concerted action of chemokines and nitric oxide. Cell Stem Cell 2, 141-150 (2008).

11. Vigo, T. et al. IFN-gamma orchestrates mesenchymal stem cell plasticity through the signal transducer and activator of transcription 1 and 3 and mammalian target of rapamycin pathways. J. Allergy Clin. Immunol. 139, 1667-1676 (2017).

12. Bai, L. et al. Hepatocyte growth factor mediates mesenchymal stem cellinduced recovery in multiple sclerosis models. Nat. Neurosci. 15, 862-870 (2012).

13. Connick, P. et al. Autologous mesenchymal stem cells for the treatment of secondary progressive multiple sclerosis: an open-label phase 2a proof-ofconcept study. Lancet Neurol. 11, 150-156 (2012).

14. Randomised double-blind placebo-controlled study of interferon beta-1a in relapsing/remitting multiple sclerosis. PRISMS (Prevention of Relapses and Disability by Interferon beta-1a Subcutaneously in Multiple Sclerosis) Study Group. Lancet 352, 1498-1504 (1998)..

15. Platanias, L. C. \& Fish, E. N. Signaling pathways activated by interferons. Exp. Hematol. 27, 1583-1592 (1999).

16. Severa, M., Rizzo, F., Giacomini, E., Salvetti, M. \& Coccia, E. M. IFN-beta and multiple sclerosis: cross-talking of immune cells and integration of immunoregulatory networks. Cytokine Growth Factor Rev. 26, 229-239 (2015).

17. Severa, M. et al. IFN-beta therapy regulates TLR7-mediated response in plasmacytoid dendritic cells of multiple sclerosis patients influencing an antiinflammatory status. J. Interferon Cytokine Res. 35, 668-681 (2015).

18. Alenda, R. et al. Blood lymphocyte subsets identify optimal responders to IFNbeta in. MS 265, 24-31 (2018).

19. Vandenbark, A. A. et al. Interferon-beta-1a treatment increases CD56 bright natural killer cells and CD4+CD25+Foxp3 expression in subjects with multiple sclerosis. J. Neuroimmunol. 215, 125-128 (2009).
20. Majchrzak-Gorecka, M., Majewski, P., Grygier, B., Murzyn, K. \& Cichy, J. Secretory leukocyte protease inhibitor (SLPI), a multifunctional protein in the host defense response. Cytokine Growth Factor Rev. https://doi.org/10.1016/j. cytogfr.2015.12.001 (2015)

21. De Rosa, $\mathrm{V}$. et al. A key role of leptin in the control of regulatory $T$ cell proliferation. Immunity 26, 241-255 (2007).

22. Kasper, L. H. \& Reder, A. T. Immunomodulatory activity of interferon-beta. Ann. Clin. Transl. Neurol. 1, 622-631 (2014).

23. Croitoru-Lamoury, J., Lamoury, F. M., Zaunders, J. J., Veas, L. A. \& Brew, B. J. Human mesenchymal stem cells constitutively express chemokines and chemokine receptors that can be upregulated by cytokines, IFN-beta, and Copaxone. J. Interferon Cytokine Res. 27, 53-64 (2007).

24. Taggart, C. C. et al. Secretory leucoprotease inhibitor binds to NF-kappaB binding sites in monocytes and inhibits p65 binding. J. Exp. Med. 202, 1659-1668 (2005).

25. Guerrieri, D. et al. Serine leucocyte proteinase inhibitor-treated monocyte inhibits human CD4(+) lymphocyte proliferation. Immunology 133, 434-441 (2011).

26. Tateosian, N. L. et al. Neutrophil elastase treated dendritic cells promote the generation of CD4(+)FOXP3(+) regulatory T cells in vitro. Cell Immunol. 269 128-134 (2011).

27. Mueller, A. M. et al. Novel role for SLPI in MOG-induced EAE revealed by spinal cord expression analysis. J. Neuroinflamm. 5, 20 (2008).

28. Norata, G. D. et al. The cellular and molecular basis of translational immunometabolism. Immunity 43, 421-434 (2015).

29. Fischer, K. et al. Inhibitory effect of tumor cell-derived lactic acid on human T cells. Blood 109, 3812-3819 (2007).

30. Dietl, K. et al. Lactic acid and acidification inhibit TNF secretion and glycolysis of human monocytes. J. Immunol. 184, 1200-1209 (2010).

31. Marin-Banasco, C. et al. Gene therapy with mesenchymal stem cells expressing IFN-ss ameliorates neuroinflammation in experimental models of multiple sclerosis. Br. J. Pharmacol. 174, 238-253 (2017).

32. Kim, M. J. et al. Effective combination of methylprednisolone and interferon beta-secreting mesenchymal stem cells in a model of multiple sclerosis. J. Neuroimmunol. 314, 81-88 (2018). 